\title{
Estudio de la difusión pulmonar de monóxido de carbono en dos situaciones clínicas: asma bronquial y diabetes mellitus
}

\author{
C. ZAMARRÓN, F. DEL CAMPO*, C. PAREDES*, J.R. RODRÍGUEZ SUÁREZ \\ Servicio de Neumología. Hospital Clínico Universitario. Santiago de Compostela. \\ *Hospital Río Ortega. Valladolid
}

\begin{abstract}
STUDY OF DIFFUSION CAPACITY OF CO IN TWO DIFFERENTS CLINICAL SITUATIONS: BRONCHIAL ASTHMA AND DIABETES MELLITUS
\end{abstract}

\begin{abstract}
RESUMEN
Objetivo: El propósito de nuestro trabajo ha sido el estudio y la comparación de la difusión pulmonar de CO (DLCO) en dos situaciones clínicas distintas, asma bronquial y diabetes mellitus.

Método: Se estudiaron 16 sujetos control, 38 pacientes con asma bronquial y 65 con diabetes mellitus. A todos ellos se les realizó un test de difusión pulmonar con $\mathrm{CO}$ por el método de la respiración única con determinación del factor de difusión de membrana (Dm) y del volumen sanguíneo capilar pulmonar (Vc).

Resultados: Hemos observado una correlación positiva significativa entre el FEV1 y la Dm. Los pacientes con asma bronquial presentan una disminución del FEV1 y del FEF25-75\% y un aumento de la DLCO, tanto de la Dm como del Vc, con respecto al grupo control. El grupo de pacientes con diabetes mellitus muestran una disminución de la CVF y del FEV1 así como de la DLCO y Vc, con respecto al grupo control. Los pacientes con asma bronquial presentan una disminución de la relación $\mathrm{Dm} / \mathrm{Vc}$ con respecto a los otros grupos.

Conclusiones: Los pacientes con asma bronquial presentan un incremento de la difusión pulmonar de monóxido de carbono, volumen sanguíneo capilar pulmonar y del factor de difusión de membrana. Por el contrario, los sujetos con diabetes mellitus muestran una disminución de la difusión pulmonar de monóxido de carbono debido, principalmente, al volumen sanguíneo capilar pulmonar.
\end{abstract}

PALABRAS CLAVE: Asma bronquial. Diabetes mellitus. Difusión pulmonar. Volumen sanguíneo capilar pulmonar.

\section{ABSTRACT}

Objective: The aim of our work has been the study and comparison of diffusion capacity of $C O(D L C O)$ in two differents clinical situations: bronchial asthma and diabetes mellitus. Method: We have studied 16 control subjects, 38 patients with bronchial asthma and 65 patients with diabetes mellitus. We performed CO pulmonary diffusion tests by single breath method to determine two components: membrane diffusion factor $(D m)$ and pulmonary capillary blood volume $(V c)$.

Results: We have found a positive correlation of FEV1 with Dm. The bronchial asthma group had a lower FEV1 and FEF25-75\% and an increase in DLCO,Dm and Vc, with respect to the control group. The diabetes mellitus group presented a decrease in CVF, FEVI, DLCO and $V c$, with respect to the control group. The bronchial asthma group showed a lower ratio of $\mathrm{Dm} / \mathrm{Vc}$ than the control and diabetes groups.

Conclusions: The bronchial asthma patients have an increase in $\mathrm{CO}$ pulmonary diffusion, membrane diffusion factor and pulmonary capillary volume. However, the diabetes mellitus patients present a decrease in $\mathrm{CO}$ pulmonary diffusion mainly due to pulmonary capillary volume.

KEY WORDS: Bronchial asthma. Diabetes mellitus. Pulmonary diffu sion tests. Pulmonary capillary blood volume.

Zamarrón C, Del Campo F, Paredes C, Rodríguez Suárez JR. Estudio de la difusión pulmonar de monóxido de carbono en dos situaciones clí nicas: asma bronquial y diabetes mellitus. An Med Interna (Madrid) 2001; 18: 237-242.

\section{INTRODUCCIÓN}

La transferencia de oxígeno entre el aire y la sangre es una función esencial del pulmón y la difusión pulmonar del monóxido de carbono (DLCO) representa una medida directa de esta capacidad. Según Roughton y Forster, la DLCO está condicionada por la resistencia de la membrana alveolo-capilar (Dm) y del volumen sanguíneo capilar pulmonar (Vc), así como por la afinidad del monóxido de carbono con la hemo- globina (1). Esta función puede verse alterada en diversas enfermedades, tanto pulmonares como extrapulmonares (2).

El asma bronquial es una enfermedad de la vía aérea caracterizada por la presencia de un incremento en la variabilidad de los flujos aéreos, pudiendo mostrar la DLCO diferentes resultados, dependiendo del grado de obstrucción bronquial $(3,4)$ e hiperinsuflación pulmonar (5). En el asma bronquial no se produce afectación primaria de la Dm o del $\mathrm{Vc}$, sino que la participación de estos factores estaría en rela-

Trabajo aceptado: 23 de Octubre de 2000

Correspondencia: Carlos Zamarrón Sanz. Magdalena 5-4A Milladoiro. 15895 La Coruña 
ción con las modificaciones de las presiones intratorácicas concomitantes a la obstrucción bronquial (3), existiendo discusión sobre cual de los componentes de la DLCO, Dm o Vc, se afecta fundamentalmente.

La diabetes mellitus se asocia a una amplia variedad de alteraciones de diferentes órganos, incluido el pulmón. En 1976, Schuyler y cols. (6) describieron la presencia de una alteración ventilatoria de tipo restrictivo en un grupo de pacientes diabéticos insulinodependientes, no fumadores y sin historia de enfermedad pulmonar previa. Posteriormente, Vracko y cols. en 1979 (7) demostraron, en estudios de microscopia electrónica, un engrosamiento de la membrana basal de los capilares pulmonares, que se ha confirmado en estudios recientes (8).

Sin embargo, el mecanismo subyacente por cual se modifica la DLCO en los pacientes con asma bronquial y diabetes mellitus continua en estudio. En este sentido, la metodología propuesta por Roughton y Forster puede ser una herramienta útil para el estudio funcional de la membrana alveolocapilar (Dm) y del volumen sanguíneo capilar pulmonar (Vc). La cuantificación de ambos factores, Dm y Vc, puede ayudarnos a explicar las alteraciones en la difusión pulmonar de gases observadas en estas dos enfermedades.

El objetivo de nuestro trabajo ha sido el estudio y comparación de la DLCO y cada uno de sus componentes, Dm y Vc, en dos situaciones clínicas distintas, asma bronquial y diabetes mellitus.

\section{MATERIAL Y MÉTODOS}

Hemos estudiado tres grupos: un grupo control y dos grupos de pacientes. El grupo control (grupo I) lo constituyen 16 sujetos sin antecedentes de enfermedad cardiopulmonar. Los grupos de estudio están formados por 38 pacientes con asma bronquial leve (grupo II) y 65 con diabetes mellitus (grupo III). Todos ellos eran no fumadores y sin alteraciones relevantes en la radiografía de tórax. En el grupo de pacientes diabéticos se registró el tiempo de evolución de la enfermedad así como la presencia de las siguientes complicaciones: neuropatía periférica, retinopatía y nefropatía. Para la identificación de estas complicaciones se realizó una exploración neurológica básica, exploración de fondo de ojo y se investigó la presencia de proteinuria $\geq 0,3 \mathrm{~g} / \mathrm{l}$, de forma repetida (9).

Se practicó un estudio de función pulmonar mediante un equipo computarizado JaegerR que consistía en una curva de flujo volumen y un test de difusión pulmonar de monóxido de carbono (DLCO) por el método de la respiración única (10) en dos fases: con oxígeno ambiental y después de respirar el paciente 5 minutos una $\mathrm{FIO}_{2}$ del $90 \%(1,11)$. Para la determinación del factor de difusión de membrana (Dm) y del volumen sanguíneo capilar pulmonar (Vc) se utilizaron las fórmulas aportadas por Roughton y Forster (1) y Cotes (11):

$$
1 / \mathrm{DLCO}=1 / \mathrm{Dm}+1 / \mathrm{q}[\mathrm{Hb}] \mathrm{Vc}
$$

donde q es la tasa de combinación del monóxido de carbono con la oxihemoglobina a una concentración dada de Hb. La variable q se calculó usando la ecuación aportada por Georges (12):

$$
1 / \mathrm{q}=0,33+0,0057 \mathrm{PCO}_{2}
$$

La determinación de la DLCO y de sus componentes Dm y Vc se repitió en tres ocasiones, en intervalos de 10 minutos, calculándose la media. Los resultados fueron corregidos para la concentración de $\mathrm{Hb}$ (13) y expresados en $\mathrm{ml} / \mathrm{min} / \mathrm{Hg}$ para la DLCO y la Dm y en ml para el Vc.

Todos los test de función pulmonar fueron realizados a temperatura ambiente siguiendo recomendaciones estándar $(14,15)$ y considerando valores anormales aquéllos que estaban por debajo del $80 \%$ de los valores teóricos.

Los parámetros de referencia para la espirometría y DLCO fueron publicados por Crapo (16) y Morris (17), respectivamente. El factor de transferencia y sus componentes son también analizados por unidad volumen de gas alveolar.

\section{Análisis estadístico}

Los datos se expresan en medias \pm DE. Para la comparación de los valores (antropométricos, espirométricos y de difusión pulmonar de $\mathrm{CO}$ ) entre los grupos a estudio se ha utilizado la prueba t de Student o el análisis de la varianza, la prueba de la U de Mann-Whitney y de Kruskal-Wallis con la corrección de Bonferroni. Para estudiar la relación entre variables continuas se ha utilizado el coeficiente de correlación de Spearman. Se consideran significativos aquellos con valores de $\mathrm{p}<0,05$.

\section{RESULTADOS}

Los datos antropométricos así como los resultados de la espirometría, obtenidos de los diferentes grupos, se exponen en la tabla I. No se observaron diferencias significativas en cuanto a la edad entre los grupos I y II, siendo los pacientes del grupo III de mayor edad que los de ambos grupos previos. Tampoco se observaron diferencias significativas entre los grupos estudiados en cuanto a la talla y el sexo. El grupo II presenta una disminución del FEV1, FEV1/FVC (\%) y del FEF $25-75 \%$ con respecto al grupo I.

Las variables obtenidas en el estudio de difusión pulmonar de $\mathrm{CO}$ en los diferentes grupos se exponen en la tabla II. El grupo II presenta un incremento del Va, DLCO, Dm, Vc y $\mathrm{Vc} / \mathrm{Va}$ con respecto al grupo I. No se observan diferencias en las variables DLCO/Va y Dm/Va entre ambos grupos. El grupo III presenta una disminución de la DLCO, DLCO/Va, Vc y $\mathrm{Vc} / \mathrm{Va}$, con respecto al grupo I. El grupo II presenta una relación $\mathrm{Dm} / \mathrm{Vc}$ significativamente menor que el grupo I y grupo III.

El grupo III está constituido por 31 (48\%) sujetos diabéticos no insulinodependientes (DNID) y 34 (52\%) insulinodependientes (DID). 16 pacientes presentaban neuropatía, 25 retinopatía y 15 nefropatía.

No existían diferencias significativas entre los grupos de DNID y de DID con respecto a los parámetros espirométricos (Tabla III), o en las variables relacionadas con la difusión pulmonar de CO (Tabla IV).

Los pacientes diabéticos con nefropatía presentaban una disminución significativa de la DLCO $(19,3 \pm 4,3 \mathrm{ml} / \mathrm{min} / \mathrm{Hg}$ vs $21,5 \pm 4,4 \mathrm{ml} / \mathrm{min} / \mathrm{Hg} ; \mathrm{p}=0,05)$ y del $\mathrm{Vc}$ con respecto al grupo $\sin$ nefropatía $(46,0 \pm 17,8 \mathrm{ml} / \mathrm{min} / \mathrm{Hg}$ vs $71,3 \pm 59,2$ $\mathrm{ml} / \mathrm{min} / \mathrm{Hg} ; \mathrm{p}=0,036)$. No se observaron diferencias con respecto a la Dm entre ambos grupos $(24,7 \pm 5,1 \mathrm{ml} / \mathrm{min} / \mathrm{Hg} v s$ 


\section{TABLA I}

CARACTERÍSTICAS ANTRO PO M ÉTRICAS Y VALORES DE LAS VARIABLES ESPIRO M ÉTRICAS DE LOS DIFERENTES GRUPOS

\begin{tabular}{|c|c|c|c|}
\hline & $\begin{array}{l}\text { Grupo I } \\
(n=16)\end{array}$ & $\begin{array}{l}\text { Grupo II } \\
(n=38)\end{array}$ & $\begin{array}{c}\text { Grupo III } \\
(n=65)\end{array}$ \\
\hline Edad (años) ${ }^{b, c}$ & $51,1 \pm 15,8$ & $51,4 \pm 11,5$ & $67,5 \pm 12,2$ \\
\hline Altura $(\mathrm{cm})^{\mathrm{ns}}$ & $163,5 \pm 2,9$ & $164,2 \pm 3,9$ & $162,3 \pm 9,3$ \\
\hline $\operatorname{Sexo} M(\%)^{n s}$ & $7(44)$ & $17(45)$ & $25(38)$ \\
\hline $\mathrm{FVC}(\mathrm{L})^{b}$ & $3,0 \pm 0,7$ & $2,8 \pm 0,7$ & $2,6 \pm 0,8$ \\
\hline $\operatorname{FVC}(\mathrm{t})(\%)^{b, c}$ & $94,6 \pm 7,8$ & $86,7 \pm 19,5$ & $64,2 \pm 24,2$ \\
\hline FEV1 (L/s) $)^{a, b, c}$ & $2,5 \pm 0,6$ & $2,2 \pm 0,6$ & $2,1 \pm 0,7$ \\
\hline FEV 1 (t) $(\%)^{b, c}$ & $102,4 \pm 12,8$ & $85,7 \pm 16,7$ & $67,8 \pm 30,0$ \\
\hline FEV1/FVC $(\%)^{\text {a }}$ & $82,7 \pm 6,8$ & $76,6 \pm 11,3$ & $81,5 \pm 14,4$ \\
\hline FEF $25-75 \%$ & $2,9 \pm$ & $2,4 \pm 0,9$ & $2,8 \pm 1,1$ \\
\hline FEF $25-75 \%$ (t) (\%) ns & $97,3 \pm 21,0$ & $81,5 \pm 27,7$ & $102,1 \pm 19,4$ \\
\hline
\end{tabular}

Grupo I: Controles. Grupo II: Pacientes con asma bronquial. Grupo III: Pacientes con diabetes mellitus.

Sexo M: Sexo masculino. FVC: Capacidad vital forzada. FVC (t) (\%): Porcentaje del valor teórico; FEV1: Volumen espiratorio forzado en el primer segundo. FEV1 (t) (\%): Porcentaje del valor teórico.

$\mathrm{FEF}_{25-75 \%}$ : Flujo espiratorio forzado entre el 25 y el $75 \%$ de la capacidad vital forzada. $\mathrm{FEF}_{25-75 \%}$ (t) (\%): Porcentaje del valor teórico.

(a) $p<0,05$ entre grupo I y grupo II

(b) $p<0,05$ entre grupo I y grupo III

(c) $p<0,05$ entre grupo II y grupo III

ns: No significativo.

\section{TABLA II}

VARIABLES O BTENIDAS EN EL ESTUDIO DE DIFUSIÓN PULM ONAR EN LOS DIFERENTES GRUPOS

\begin{tabular}{|c|c|c|c|}
\hline & $\begin{array}{l}\text { Grupo I } \\
(n=16)\end{array}$ & $\begin{array}{l}\text { Grupo II } \\
(n=38)\end{array}$ & $\begin{array}{c}\text { Grupo III } \\
(n=65)\end{array}$ \\
\hline VA (L) $)^{a, c}$ & $4,1 \pm 1,0$ & $5,0 \pm 1,5$ & $4,2 \pm 1,1$ \\
\hline $\mathrm{DLCO}(\mathrm{ml} / \mathrm{min} / \mathrm{Hg})^{a, b, c}$ & 24,1 & 31,2 & $21,6 \pm 4,9$ \\
\hline DLCO (t) $(\%)^{a, c}$ & $95,5 \pm 11,5$ & $119,6 \pm 24,4$ & $96,2 \pm 7,4$ \\
\hline DLCO/VA ${ }^{\mathrm{b}, \mathrm{c}}$ & $5,9 \pm 0,8$ & 6,3 & $5,6 \pm 1,1$ \\
\hline $\mathrm{Dm}(\mathrm{ml} / \mathrm{min} / \mathrm{Hg})^{a, c}$ & $27,8 \pm 7,1$ & $34,0 \pm$ & $25,2 \pm 5,3$ \\
\hline $\mathrm{Dm} / \mathrm{VA}^{\mathrm{c}}$ & $6,7 \pm 1,4$ & $7,1 \pm 2,1$ & $6,3 \pm 1,1$ \\
\hline$V_{c}(m l){ }^{a, b, c}$ & $104,1 \pm 121,3$ & $206,0 \pm 118,0$ & $57,6 \pm 32,0$ \\
\hline$V c / V A a, b, c$ & $32,3 \pm 49,8$ & $51,2 \pm 41,3$ & $14,6 \pm 9,3$ \\
\hline $\mathrm{Dm} / \mathrm{Vc}^{\mathrm{a}, \mathrm{b}, \mathrm{c}}$ & $0,46 \pm 0,2$ & $0,23 \pm 0,17$ & $0,60 \pm 0,3$ \\
\hline
\end{tabular}

Grupo I: Controles. Grupo II: Pacientes con asma bronquial. Grupo III: Pacientes con diabetes mellitus.

VA: Volumen alveolar. DLCO : Difusión pulmonar de CO. DLCO (t) (\%): Porcentaje del valor teórico $\mathrm{V} c$ : Volumen sanguíneo capilar pulmonar. Dm: Difusión pulmonar de membrana.
(a) $p<0,05$ entre grupo I y grupo II
(b) $p<0,05$ entre grupo I y grupo III
(c) $p<0,05$ entre grupo II y grupo III
ns: No significativo.

$27,1 \pm 7,4 \mathrm{ml} / \mathrm{min} / \mathrm{Hg} ; \mathrm{p}=\mathrm{NS})$. Tampoco se observaron diferencias entre los sujetos diabéticos con neuropatía o retinopatía con respecto a los pacientes sin estas complicaciones.

Existe una correlación positiva significativa entre $\mathrm{FEV}_{1}$ con la DLCO ( $\mathrm{r}=0,33 ; \mathrm{p}=0,01)$ (Fig. 1) y con la Dm ( $\mathrm{r}=$ $0.42 ; \mathrm{p}=0,001)$ (Fig. 2). No se observa correlación entre el $\mathrm{FEV}_{1}$ y el $\mathrm{Vc}(\mathrm{r}=0.15 ; \mathrm{p}=\mathrm{NS})$.
TABLA III

CARACTERÍSTICAS ANTRO PO MÉTRICAS Y VALORES DE LAS VARIABLES ESPIRO M ÉTRICAS DE LOS PACIENTES DIABÉTICOS

\begin{tabular}{|c|c|c|c|}
\hline & $\begin{array}{c}\text { DNID } \\
(n=31)\end{array}$ & $\begin{array}{c}\text { DID } \\
(n=34)\end{array}$ & $p$ \\
\hline Edad (años) & $71,1 \pm 9,2$ & $66,5 \pm 13,2$ & ns \\
\hline Altura $(\mathrm{cm})$ & $160,0 \pm 8,4$ & $163,8 \pm 10,3$ & ns \\
\hline Sexo $M(\%)$ & $5(31)$ & $17(45)$ & ns \\
\hline $\mathrm{FVC}(\mathrm{L})$ & $2,5 \pm 0,7$ & $2,6 \pm 0,8$ & ns \\
\hline FVC (t) $(\%)$ & $58,7 \pm 22,4$ & $65,0 \pm 25,2$ & ns \\
\hline FEV1 (L/S) & $2,0 \pm 0,7$ & $2,1 \pm 0,6$ & ns \\
\hline FEV $1(t)(\%)$ & $62,7 \pm 29,3$ & $71,6 \pm 30,6$ & ns \\
\hline FEV 1/FVC (\%) & $80,9 \pm 17,5$ & $83,1 \pm 13,9$ & ns \\
\hline FEF $_{25-75 \%}$ & $2,6 \pm 1,0$ & $2,9 \pm 1,2$ & ns \\
\hline $\operatorname{FEF}_{25-75 \%}(\mathrm{t})(\%)$ & $100,5 \pm 20,3$ & $103,2 \pm 18,5$ & ns \\
\hline
\end{tabular}

DNID: Pacientes con diabetes no insulinodependiente. DID: Pacientes con diabetes insulinodependiente.

ns: No significativo.

FVC: Capacidad vital forzada. FVC (t) (\%): Porcentaje del valor teórico: FEV1: Volumen espiratorio forzado en el primer segundo. FEV1 (t) (\%): Porcentaje del valor teórico. $\mathrm{FEF}_{25-75 \%}$ : Flujo espiratorio forzado entre el 25 y el 75\% de la capacidad vital forzada. FEF $_{25-75 \%}$ (t) (\%): Porcentaje del valor teórico.

TABLA IV

VARIABLES O BTENIDAS EN EL ESTUDIO DE DIFUSIÓN PULM O NAR EN LOS PACIENTES DIABÉTICOS

\begin{tabular}{lccc}
\hline & $\begin{array}{c}\text { DNID } \\
(n=31)\end{array}$ & $\begin{array}{c}\text { DID } \\
(n=34)\end{array}$ & $p$ \\
\hline VA (L) & $4,1 \pm 0,9$ & $4,3 \pm 1,2$ & $n s$ \\
DLCO $(\mathrm{ml} / \mathrm{min} / \mathrm{Hg})$ & $21,5 \pm 4,6$ & $20,7 \pm 5,2$ & $\mathrm{~ns}$ \\
DLCO $(\mathrm{t})(\%)$ & $95,2 \pm 6,7$ & $96,3 \pm 8,0$ & $\mathrm{~ns}$ \\
DLCO/VA & $5,5 \pm 1,4$ & $5,6 \pm 1,1$ & $\mathrm{~ns}$ \\
Dm $(\mathrm{ml} / \mathrm{min} / \mathrm{Hg})$ & $26,0 \pm 5,7$ & $24,8 \pm 5,9$ & $\mathrm{~ns}$ \\
Dm/VA & $6,2 \pm 0,8$ & $6,2 \pm 1,3$ & $\mathrm{~ns}$ \\
Vc (ml) & $70,7 \pm 71,9$ & $61,2 \pm 32,4$ & $\mathrm{~ns}$ \\
Vc/VA & $18,7 \pm 21,8$ & $15,5 \pm 9,4$ & $\mathrm{~ns}$ \\
Dm/Vc & $0,69 \pm 0,32$ & $0,56 \pm 0,32$ & $\mathrm{~ns}$
\end{tabular}

DNID: Pacientes con diabetes no insulinodependiente. DID: Pacientes con diabetes insulinodependiente.

ns: No significativo.

VA: Volumen alveolar. DLCO : Difusión pulmonar de CO. DLCO (t) (\%): Porcentaje del valor teórico Dm: Difusión pulmonar de membrana.Vc: Volumen sanguíneo capilar pulmonar.

\section{DISCUSIÓN}

En este estudio se observa que los pacientes asmáticos presentan un incremento de la difusión pulmonar de monóxido de carbono, así como de los componentes factor de difusión de membrana y volumen sanguíneo capilar pulmonar. En los pacientes diabéticos se corrobora la presencia de un trastorno restrictivo. Además observamos una disminución de la DLCO y del volumen capilar, que es más marcado en los pacientes con nefropatía. 


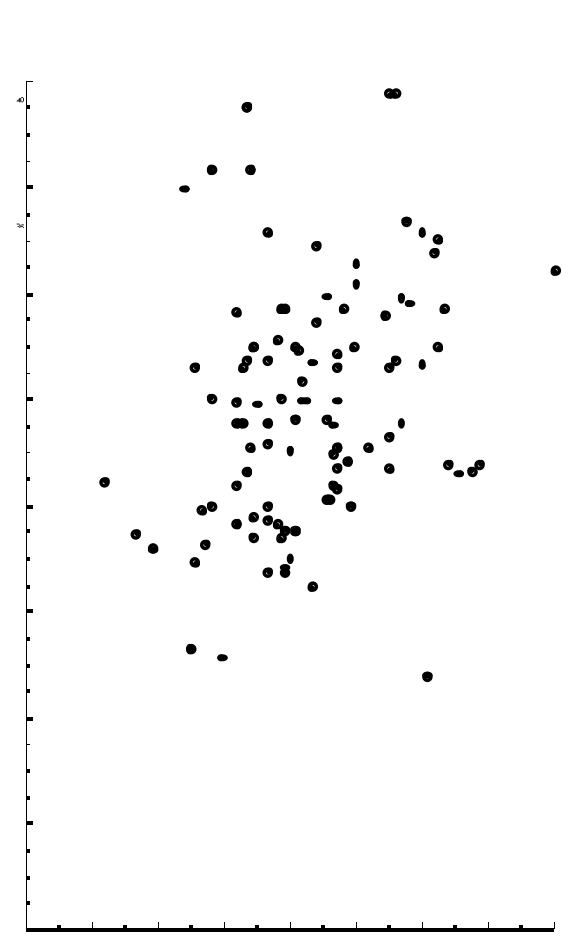

Fig. 1. Correlación significativa entre la DLCO $(\mathrm{ml} / \mathrm{min} / \mathrm{Hg})$ y $\mathrm{FEV}_{1}$ (L/S).

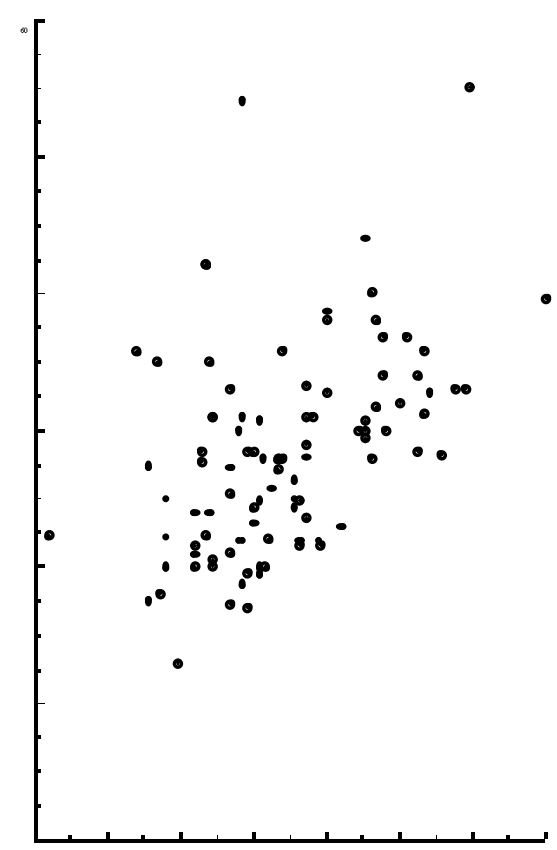

Fig. 2. Correlación significativa entre la $\mathrm{Dm}(\mathrm{ml} / \mathrm{min} / \mathrm{Hg})$ y $\mathrm{FEV}_{1}$ (L/s).
Utilizando el método de Roughton y Forster (1), hemos cuantificado la Dm y el Vc en dos modelos clínicos distintos. Es conocido que en el asma bronquial no se produce afectación primaria de la Dm o del Vc, sino que las modificaciones de estos factores se deberían a los cambios en las presiones intratorácicas (3-5). Sin embargo, la diabetes mellitus puede afectar de forma directa a los factores implicados en la difusión pulmonar $(7,8)$.

Diferentes estudios han mostrado un incremento de la DLCO en sujetos asmáticos $(3,4,18$-20) el cual está asociado a la presencia de obstrucción en la vía aérea (18) y que evoluciona hacia la normalidad cuando se instaura tratamiento broncodilatador $(3,19,21)$. Este aumento en la DLCO se debe tanto al Vc como a la Dm.

En el asma bronquial, la obstrucción inspiratoria de las vías aéreas incrementa la negatividad de la presión intratorácica (3), favoreciendo el recrutamiento de capilares a nivel de las zonas apicales del pulmón y aumentando el volumen capilar pulmonar $(22,23)$. Este fenómeno también ha sido observado en otras circunstancias experimentales $(24,25)$ y tiene como objetivo optimizar el intercambio gaseoso (23). Por otra parte, la hiperinsuflación pulmonar, también presente en algunos sujetos asmáticos, favorecería el recrutamiento de alvéolos previamente cerrados, distendiendo la membrana alveolocapilar y agrandando la Dm (26).

En nuestro estudio, los pacientes asmáticos presentan un incremento de la DLCO, Dm y Vc, así como del volumen alveolar. El aumento de la Dm se corrige con el volumen alveolar, lo que sugiere su dependencia de la hiperinsuflación pulmonar. Esta asociación también se apoya por el hallazgo de una correlación positiva entre el $\mathrm{FEV}_{1}$ y la Dm. En cambio, el incremento del Vc observado en el grupo de pacientes con asma bronquial no se normaliza con el volumen alveolar ni muestra correlación significativa con el $\mathrm{FEV}_{1}$. No obstante, en algunos estudios, este parámetro de la difusión pulmonar se muestra muy sensible al empeoramiento del asma (27), aunque no dependa directamente de los cambios en el volumen pulmonar de aire (28).

Existe evidencia de que la diabetes mellitus puede afectar al sistema respiratorio, condicionando alteraciones en la función pulmonar (29-31). En este estudio, se corrobora que los pacientes diabéticos presentan un alteración ventilatoria de tipo restrictivo (6,29-32), que se asocia al control clínico de la enfermedad $(33,34)$. También se ha descrito una disminución de la DLCO y del Vc (35-39), pero sin modificaciones de la Dm $(35,36)$. Todo ello apoya la idea de que la lesión estructural de la membrana basal del capilar pulmonar, descrita en estos enfermos $(7,8)$, tiene poca expresión funcional (39). En nuestro estudio los pacientes diabéticos presentan una disminución del Vc y de la Dm con respecto al grupo control, aunque el último factor no alcanzó significación estadística. Por tanto, el Vc parece estar más implicado que la Dm en la disminución de la DLCO en este grupo de enfermos $(35,36)$.

En los sujetos asmáticos, la presencia de una relación $\mathrm{Dm} / \mathrm{Vc}$ menor que la de los controles y diabéticos puede explicarse, esencialmente, por un aumento del Vc. En cambio, el incremento de esta relación observado en sujetos con diabetes mellitus puede atribuirse a la disminución de los valores del mismo parámetro en estos pacientes. En definitiva, en ausencia de modificaciones en los flujos aéreos, el volumen sanguíneo capilar pulmonar parece ser el principal factor relacionado con los cambios en la DLCO tanto en el asma bronquial como en la diabetes mellitus. 
Entre las complicaciones vasculares asociadas a la diabetes mellitus, las manifestaciones renales y retinianas se han estudiado frecuentemente $(40,41)$. También se han demostrado en otros órganos lesiones asociadas a la presencia de microangiopatía diabética (42), incluido el pulmón (43).

La relación entre las complicaciones vasculares diabéticas y los trastornos en la difusión pulmonar de estos enfermos, han sido objeto de controversia (31,37). En 1988 Briton estudió la DLCO en 20 sujetos con retinopatía diabética y 20 sin esta complicación, no observando diferencias entre ambos grupos (44). Sin embargo, en otros estudios posteriores, se ha demostrado una disminución de la DLCO en diabéticos con retinopatía (45) o con otras complicaciones vasculares (4648), sugiriendo una asociación entre la microangiopatía diabética y las anomalías de la difusión pulmonar observadas en estos pacientes. En nuestro estudio, los diabéticos con complicaciones renales presentaron una disminución del volumen capilar pulmonar con respecto al grupo sin esta complicación. No se han observado alteraciones de la función pulmonar asociadas a la presencia de neuropatía o retinopatía. Tampoco se han hallado diferencias entre pacientes insulinodependientes y no insulindependientes.
En algunos estudios, se ha encontrado una asociación significativa entre años de evolución de la diabetes y la presencia de alteraciones funcionales pulmonares $(35,45)$, aunque en otros trabajos ha sido cuestionada $(6,49)$. Nosotros no hemos encontrado relación entre ambas variables.

En resumen, los pacientes con asma bronquial presentan un incremento de la difusión pulmonar de monóxido de carbono y de sus dos componentes, volumen sanguíneo capilar pulmonar y del factor de difusión de membrana. Este último se asocia a la presencia de obstrucción bronquial. Por el contrario, los sujetos con diabetes mellitus muestran una disminución de la difusión pulmonar de monóxido de carbono y del volumen sanguíneo capilar pulmonar, más acentuado en los sujetos con nefropatía, lo cual podría reflejar la presencia de microangiopatía a nivel pulmonar.

\section{AGRADECIMIENTOS}

Los autores expresan su agradecimiento a F. Gude (Unidad de Epidemiología Clínica del Hospital Clinico Universitario) por la ayuda prestada en el análisis de los datos.

\section{Bibliografía}

1. Roughton FJW, Forster RE. Relative importance of diffusion and chemical reaction rates in determining rate of exchange of gases in the human lung with special reference to true diffusing capacity of pulmonary membrane and volume of blood in the lung capillaries. J Appl Physiol 1957; 11: 290-302.

2. Cotes JE. Gas exchange for carbon monoxide. In: Lung function. Assessment and application in medicine. 5th ed. Oxford: Blackwell Scientific Publications 1993

3. Keens TG, Mansell A, Krastins IRB, Levison H, Bryan AC, Hyland $\mathrm{RH}$, Zamel N. Evaluation of the single breath diffusing capacity in asthma and cystic fibrosis. Chest 1979; 76: 41- 44.

4. Cotton DJ, Soparkar GR, Graham BL. Diffusing capacity in the clinical assessment of chronic airflow limitation. Med Clin North Am 1996; 80: 549-564.

5. Pecora LJ, Berstein IL, Feldman DP. Pulmonary diffusion capacity, membrane diffusing capacity and capillary blood volume in children with intractable asthma with and without chronic overinflation of the lungs. J Allergy 1966; 37: 204-215.

6. Schuyler MR, Niewoehner DE, Inkley SR, Kohn R. Abnormal lung elasticity in juvenile onset diabetes mellitus. Am Rev Respir Dis 1976; 113: $37-41$.

7. Vracko R, Thorning D, Huang TW. Basal lamina of alveolar epithelium and capillaries: quantitative changes with ageing in diabetes mellitus. Am Rev Respir Dis 1979; 120: 973-983.

8. Weynand B, Jonckheere A, Frans A, Rahier J. La diabetes mellitus induce un engrosamiento de la lámina basal pulmonar. Respiration (Ed. esp) 1999; 3: 168-173.

9. Nishimura M, Miyamoto K, Suzuki A, Yamamoto H, Tusuji M, Kishi F, Kawakami Y. Ventilatory and heart rate responses to hypoxia and hypercapmia in patients with diabetes mellitus. Thorax 1989; 44: 251-257.

10. Ogilvie CM, Forster RE, Blakemore WS, Morton JW. A standardised breatholding technique for the clinical measurement of diffusing capacity in man. J Physiol (London) 1959; 146: 572-582.

11. Cotes JE. Measurement of transfer factor (Diffusing capacity) and its subdivisions. In: Lung function. Assessment and application in medicine. 5th ed. Oxford: Blackwell Scientific Publications 1993.

12. Georges R, Saumon S, Loiseau A. The relationship of age to pulmonary membrane conductance and capillary blood volume. Am Rev Respir Dis 1978; 117: 1069-1078.

13. Crapo RO. Carbon monoxide diffusing capacity (transfer factor). Semi- nars in Respiratory and Critical Care Medicine 1998; 19: 335-347.

14. American Thoracic Society. Standardization of spirometry: 1987 update. Am Rev Resp Dis 1987; 136: 1285-1298.

15. American Thoracic Society. 1987. Single breath carbon monoxide diffusing capacity (transfer factor). Recommendations for standard technique. Am Rev Resp Dis 136: 1299- 1307.

16. Crapo RO, AH Morris, Gardber RM. Reference spirometric values using techniques and equipment that meet ATS recommendations. Am Rev Respir Dis 1981;123: 659-664.

17. Morris JF, Koski A, Johnson LC. Spirometric standards for healthy nonsmoking adults. Am Rev Respir Dis 1971;103: 57-67.

18. Stewart RI. Carbon Monoxide diffusing capacity in asthmatic patients with mild airflow limitation. Chest 1988; 94: 332-336.

19. Knudson RJ, Kalternorn WT, Burrows B. Single breath carbon monoxide transfer factor in different forms of chronic airflow obstruction in a general population sample. Thorax 1990; 45: 514-519.

20. Viegi G, Paoletti P, Carrozi L, Baldacci S, Modena P, Pedreschi M, Di Pede F, Mammini U, Giuntini C. CO diffusing capacity in a general population sample: relationships with cigarette smoking and airflow obstruction. Respiration 1993; 60: 155-161.

21. Gazioglu K, Condemi JJ, Hyde RW, Kaltreimer NL. Effect of isoproterenol on gas exchange during air and oxygen breathing in patients with asthma. Am J Med 1971; 50:185-190.

22. Weinberger SE, Johnson TS, Weiss ST. Clinical significance of pulmonary function tests. Use and interpretation of a single breath diffusing capacity. Chest 1980; 78: 483-489.

23. Collard P, Njinou B, Nejadnik B, Keyeux A, Frans A. Single breath diffusing capacity for carbon monoxide in stable asthma. Chest 1994; 105 : 1426-1429.

24. Hopkins SR, Belzberg AS, Wiggs BR, Mckenzie DC. Pulmonary transit time and diffusion limitation during heavy exercise in athletes. Respiration Physiol 1996; 103: 67-73.

25. Vaida P, Kays C, Riviere D, Techoueyres P, Lachaud JL. Pulmonary diffusing capacity and pulmonary capillary blood volume during parabolic flights. J Appl Physiol 1997; 82: 1091-1997.

26. Werner F, Kolmer B. The CO single Breath transfer factor of the lung. Generally acceptable normal values. Pflügers Arch 1982; 393: 269-270.

27. Desjardín JA, Sutarik JM, Suh BY, Ballard RD. Influence of sleep on pulmonary capillary volume in normal and asthmatic subjects. Am J Respir Crit Care Med 1995; 152: 193-198. 
28. Gurtner GH, Fowler WS. Interrelationsships of factors affecting pulmonary diffusing capacity. J Appl Physiol 1971; 30: 619-624.

29. Lange P, Groth S, Kastrup J, Mortensen J, Appleyard M, Nyboe J, Schnohr P, Jensen G. Diabetes mellitus, plasma glucose and lung function in a cross-sectional population study. Eur Respir J 1989; 2: 14-19.

30. Lange P, Groth S, Mortensen J, Appleyard M, Nyboe J, Schnohr P, Jensen G. Diabetes mellitus and ventilatory capacity; a five year follow-up study. Eur Respir J 1990; 3: 288-292.

31. Sandler M. Is the lung a "target organ" in diabetes mellitus?. Arch Intern Med 1990; 150: 135-1358.

32. Ramirez LC, Nogare AD, Hsia C, Arauz C, Butt Y, Strowig, Schnurr Breen L, Raskin P. Relationship between diabetes control and pulmonary function in insulin dependent diabetes mellitus. Am J Med 1991; 91: 371-373.

33. Bell D, Collier A, Matthews DM, Cooksey EJ, Ross Mchardy GJ, Clarke BF. Are reduced lung volumes in IDDM due to defect in connective tissue?. Diabetes 1988, 37: 829-831.

34. Okubo MH, Okamura M, Yamane K, Kado S, Egusa G, Hiramoto T, Hara H, Yamakido M. Abnormalities of pulmonary function in patients with non insulin dependent diabetes mellitus. Intern Med 1992; 31: 189193.

35. Sandler M, Bunn AE, Stewart RI. Pulmonary function in young insulin dependent diabetics. Chest 1986; 90: 670-675.

36. Sandler M, Bunn AE, Stewart Y. Cross sectional study of pulmonary function in patients with insulin dependent diabetes mellitus. Am Rev Respir Dis 1987, 135: 223-229.

37. Cooper BG, Taylor R, Alberti KG, Gibson GJ. Lung function in patients with diabetes mellitus. Respir Med 1990; 84: 235-239.

38. Mancini M, Filippelli M, Seghieri G, Iandelli I, Innocenti F, Duranti R, Scano G. Respiratory muscle function and hypoxic ventilatory control in patients with type I diabetes. Chest 1999; 115: 1553-1562.

39. Frans A, Jonkheere A, Minette PH, Buyschaert M, Lambert A, Rahier J. Normal function of the pulmonary bed with thickened basal membrane in diabetes mellitus. Am Rev Respir Dis 1998; 137 (4) Suppl 2: 272.

40. Berg TJ, Bangstad HJ, Torjesen PA, Osterby R, Bucala R, Hanssen KF.
Advanced glycation end products in serum predict changes in the kidney morphology of patients with insulin-dependent diabetes mellitus. Metabolism 1997; 46: 661-665.

41. Lu M, Kuroki M, Amano S, Tolentino M, Keough K, Kimm I, Bucala $\mathrm{R}$, Adamis AP. Advanced glycation end products increase retinal vascular endothelial growth factor expression. J Clin Invest 1998; 101: 12191224.

42. Sims TJ, Rasmussen LM, Oxlund H, Baile AL. The role of glycation cross-links in diabetic vascular stiffening. Diabetologia 1996; 39: 946951.

43. Popov D, Simionescu M. Alterations of lung structure in experimental diabetes, and diabetes associated with hyperlipidemia in hamsters. Eur Respir J 1997; 10: 1850-1858.

44. Briton J. Is the carbon monoxide transfer factor diminished in the presence of diabetic retinopathy in patients with insulin dependent diabetes mellitus?. Eur Respir J 1988; 1: 403-406.

45. Isotani H, Nakamura Y, Kameoka K, Tanaka K, Furukawa K, Kitaoka $\mathrm{H}$, Ohsawa. Pulmonary diffusing capacity, serum angiotensin converting enzyme activity and angiotensin convertin enzyme gene in Japanese non insulin dependent diabetes mellitus patient. Diabetes Research and Clinical Practice 1999; 43: 173-177.

46. Weir DC, Jennings P, Hendy M, Barnett A, Burge PS. Transfer factor for carbon monoxide in patients with diabetes with and without microangiopathy. Thorax 1988; 43: 725-726.

47. Mori H, Okubo M, Okamura M, Yamane K, Kado S, Egusa G, Hiramoto T, Hara H, Yamakido M. Abnormalities of pulmonary function in patients with non-insulin-dependent diabetes mellitus. Intern Med 1992;31:189-193.

48. Scnack Ch, Festa A, Schwarzmaier-Dássié, Haber P, Schernthaner. Pulmonary dysfunction in type 1 diabetes in relation to metabolic long term control and to incipient diabetic nefropathy. Nephron 1996; 74 : 395-400.

49. Oulhen P, Barthélémy L, Bellet-barthas M, Darragon T. Contribution a l'étude de la fonction respiratoire du diabétique insulino-dépendant. Rev fr Mal Resp 1982; 10: 213-224. 